\title{
Space Rocks and Optimizing Scanning Electron Channeling Contrast
}

\author{
T. Ben Britton ${ }^{1}$, Daniel Goran ${ }^{2}$ and Vivian S. Tong ${ }^{1,3}$ \\ 1. Department of Materials, Imperial College London, UK. \\ 2. Bruker Nano GmbH, Germany. \\ 3. Now at NPL, Teddington, UK.
}

In the scanning electron microscope, all electrons are known to channel when incident on crystalline materials. In the present work, we explore methods to optimize the image contrast for electron channeling contrast images captured using forescatter diodes (FSD). Our optimization approach utilizes systematic exploration of the detector capture angle and incident sample geometry and repeat imaging of the same region from a sample of the Gibeon meteorite. We compare these polycrystalline ECCI micrographs from the different capture conditions also with electron backscatter diffraction (EBSD) pattern analysis. The sample was tilted to $70^{\circ}$, as used for conventional EBSD based analysis.

EBSD analysis reveals the presence of the FCC taenite (austenite like) and BCC kamacite (ferrite like) phases. For the region scanned, the EBSD mapping reveals a strong orientation relationship where all the kamacite orientations belong to one parent taenite orientation. There are several variants of the kamacite phase present within the scanned area.

Energy dispersive X-ray spectroscopic (EDX/EDS) analysis reveals that the FCC phase is Ni rich and the BCC phase is Ni poor. Cluster based segmentation has been used to provide sum-based characteristic spectra of these two phases.

The angle at which the FSD were presented with respect to the sample were changed, by tilting the EBSD camera that they were attached to. It was found that a shallow take off angle for the electrons which strike the FSD diodes emphasized surface topography contrast. Black-white contrast can be interpreted to understand relief on the surface of the sample. RGB 'plume' contrast, created due to the occlusion of exit beams which could strike the three FSD diodes (false coloured to Red, Green and Blue channels [2]), can be used to determine if there are protrusions on the surface (e.g. due to colloidal silica).

The angle subtended by the FSD diodes was altered by retracting the EBSD camera from the sample. When the detector was positioned to optimize orientation contrast, the orientation contrast was maximized, as compared to the topographic signal, when the detector was positioned further back.

After positioning the detector for obtaining the best orientation contrast, the sample was rotated around the sample surface normal in $0.1^{\circ}$ increments. This changed the contrast in the FSD micrographs and revealed the presence of subtle sub-grains within the slow cooled meteorite sample.

The nature of contrast in FSD based ECCI micrographs is discussed in terms of channeling-in vs channeling-out [3]. To assess these two signals, in the present work the EBSD patterns were used to generate virtual FSD images. The contrast of the FSD images could be replicated using just the background signal (obtained via fitting a Gaussian function to the 2D EBSD pattern). Contrast could also be obtained using the background corrected EBSD patterns, revealing that there is contrast also 
from the channeling out signal, but the signal to noise ratio for the background signal is much less than the channeling out analysis. This indicates that the most substantive contrast contribution is from the channeling-in of electrons.

Our systematic approach provides method to optimize the contrast from FSD based imaging and improves the robustness of micrograph analysis for SEM based ECCI based imaging [4].

\section{References:}

[1] AJ Wilkinson and TB Britton, Materials Today (2012).

[2] AP Day and TE Quested, J. Microsc. (1999).

[3] A Winklemann et al., J. Microsc. (2017).

[4] TBB and VT acknowledge Imperial Innovations and HEFCE for funding under joint proof of concept funding. TBB acknowledges funding for his research fellowship from the Royal Academy of Engineering. We thank Aimo Winkelmann and Gert Nolze for helpful discussions in interpreting this data and the virtual imaging modes. The basis of this work is published Britton, T.B. et al. J. Mater. Char (2018) and as a preprint https://arxiv.org/abs/1804.08754

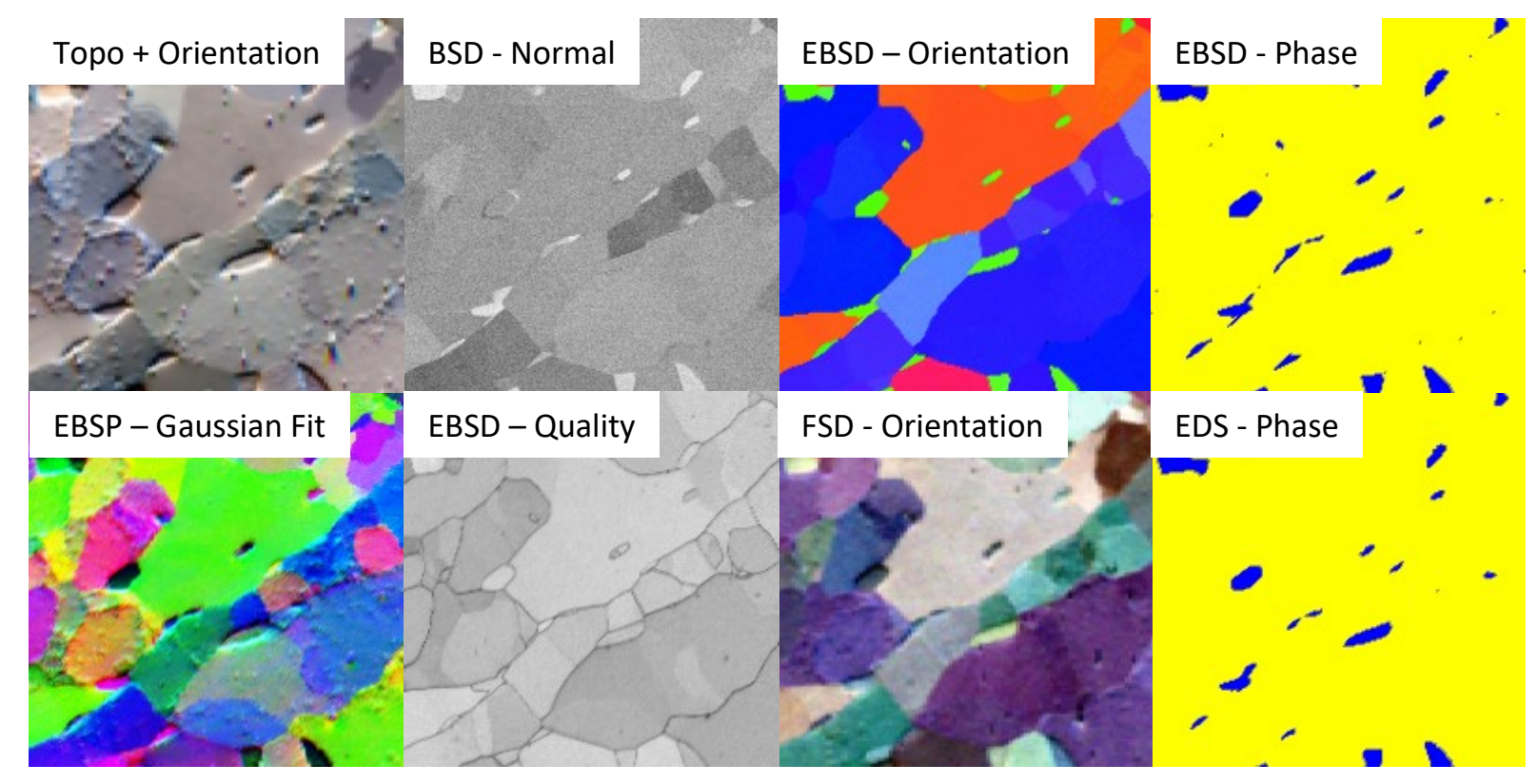

Figure 1. Analysis of the obtained from microstructural imaging of a region of the Gibeon meteorite using FSD, EBSD, EDS imaging techniques. 